\title{
Research on Set Theory Based on Paraconsistent Logic
}

\author{
Shi Jing \\ School of Culture and Media, Central University of Finance and Economics, Beijing, China \\ Email address: \\ shijing0707@163.com \\ To cite this article: \\ Shi Jing. Research on Set Theory Based on Paraconsistent Logic. International Journal of Philosophy. Vol. 8, No. 2, 2020 , pp. 43-48. \\ doi: $10.11648 /$ j.ijp.20200802.13
}

Received: May 1, 2020; Accepted: May 25, 2020; Published: June 3, 2020

\begin{abstract}
Different from ZF axiomatic set theory, the paraconsistent set theory has changed the basic logic of set theory and selected paraconsistent logic which can accommodate or deal with contradictions, it effectively avoids the whole theory falling into a non-trivial dilemma when there are contradictions in set theory. In this paper, we first review the history and current situation of the praconsistent set theory; then, we give three kinds of paraconsistent logic which can be used to construct the praconsistent set theory among many kinds of paraconsistent logics. And then, we analyze the differences of methods of the paraconsistent set theory with strong or weak structure of paraconsistent logic and get different paraconsistent set theory. Finally, we verify that paraconsistent set theory is a new method to solve the paradox of set theor. The development of paraconsistent set theory can solve the difficulties in the development of set theory in a unique way, which is not only the extension of the application of paraconsistent logic, but also the new form and new trend of the development of set theory.
\end{abstract}

Keywords: Set Theory, Paraconsistent Logic, Paradox

\section{Introduction}

Cantor established the naive set theory with the principles of comprehension and extension. The establishment of Cantor's naive set theory (hereafter referred to as the set theory) has brought new vitality to mathematics. It has produced two fields of modern mathematics: meta-mathematics and structural mathematics, which are completely different from the classical mathematics. At the beginning of the 20th century, Russell discovered the contradiction in the set theory, that is, the familiar Russell paradox, which triggered the third crisis in mathematics, and everyone debated "What does the set mean?". At that time, many scholars believed that only by eliminating the paradox can the set theory be saved. Among the various solutions proposed, Zemelo adopted Hilbert's axiomatic method in 1908 to limit the general principles in the set theory to avoid paradoxes, which was an earlier and more effective way. Zemelo regards the set theory as an abstract axiomatization theory. The properties of sets are characterized by axioms, and sets are not defined here. This theory was later improved by Fraenkel and Skolem and others to become the current ZF axiomatic set theory (Zemelo- Fraenkel set theory), referred to as ZF set theory for short. ZF axiomatic set theory is a formal system formed by adding non-logical axioms about the basic properties of sets to the classic logic with "=" and " $\in$ ". If you add the choice axiom AC, you get ZFC. Therefore, ZF axiomatic set theory can be simply regarded as an axiomatic set theory that adds non-logical axioms on the basis of classical logic.

After 1960s, many scholars put forward a series of questions in the framework of non classical logic, such as: "Is the set theory consistent?" Can non classical logic save the set theory? Is there a new solution to the inconsistency of comprehension principle in the set theory? In the face of these problems, there is an upsurge of rescuing the set theory with the non classical logic as the basic logic [1]. In that period, the set theory of paraconsistency was born, which was based on the set theory of Philosophical Logic.

In the classical axiomatic set theory system ZFC, subset axiom is a kind of restriction to the comprehension principle of the set theory, which excludes Russell paradox in the set theory. Paraconsistent set theory is different from ZF's axiomatic set theory. ZF adopts the method of restricting sets by axioms. The study of paraconsistent sets began in the 1960s, which is a logic that has changed the basic logic of the set theory, and has chosen the paraconsistent logic that can accommodate or deal with contradictions, and thus effectively avoids the contradictions in the set theory and makes the 
whole theory fall into a non-trivial dilemma. Although the classical axiomatic set theory dispels the paradox, it changes Cantor's original assumption, while the paraconsistent set theory retains two principles of the set theory. In this paper, we first review the history and research status of the paraconsistent set theory; then, we give three kinds of paraconsistent logics which can be used to construct the paraconsistent set theory; then, we analyze the different characteristics of the paraconsistent set theory constructed by strong or weak paraconsistent logic; finally, we verify that the paraconsistent set theory is a new method to solve the paradox of the set theory.

\section{The History and Research Status of Paraconsistent Set Theory}

In 1963, Da Costa, based on the generation of paraconsistent set theory of paraconsistent logic $\mathrm{C}_{\mathrm{n}}{ }^{=}(1 \leq \mathrm{n}$ $\leq \omega)$, constructed the system of paraconsistent set theory as a sign in Calculs propositionnels pour les systemes formels inconsistants. In 1964, A. I. Arruda and da Costa firstly defined the formal system hierarchy: $\mathrm{NF}_{0}, \mathrm{NF}_{1}, \ldots, \mathrm{NF}_{\mathrm{n}}, \ldots$, $\mathrm{NF}_{\omega}$, in A hierarchy of formal systems, and studied the main characteristics of the systems. Secondly, after strengthening the system $\mathrm{NF}_{\mathrm{i}}(\mathrm{i} \geq 1)$, some important conclusions of $\mathrm{NF}_{0}$ are obtained. Thirdly, the incompatibility and undecidability of these systems are described [2]. In 1982, A. I. Arruda and D. Batens constructed the system of the paraconsistent set theory with Russell's set $\mathrm{R}$ in Russell's set versus the universal set in paraconsistent set theory, and proved that $\cup \mathrm{R}$ is a complete set in the strong paraconsistent set theory with Russell's set (that is, $\mathrm{R}=\{\mathrm{x}: \mathrm{x} \notin \mathrm{X}\}$ ). First of all, the paper points out that one of them has proved in the previous paper that UUR is a complete set in the paraconsistent set theory of Da Costa with Russell's set $\mathrm{R}=\{\mathrm{x}: \mathrm{x} \notin \mathrm{x}\}$. Then it is pointed out that, in this paper, we prove that this conclusion is valid in all the "strong" paraconsistent set theories (especially in all the paraconsistent set theories of Da Costa) [3]. In 1985, in the paper Remarks on da Costa's paraconsistent set theories. Rev Colombiana Mat, 1985, 19 (1-2): 9-24, A. Arruda constructed the set theory based on the paraconsistent set theory of Da Costa, and took Quine's set theory NF as the base and then proved that UUR was a complete set in any paraconsistent set theory with Rusell's set R. And the existence of Russell's set is incompatible with the modes of separation axiom and substitution axiom. In the set theory of any Da Costa with type $\mathrm{NF}$, the mode of comprehension axiom must be expressed precisely in NF. Otherwise, some paradoxes can be deduced to make the theory invalid [4]. In 1986, in the research paper On paraconsistent set theory. Logique et Analyse, Da Costa constructed the paraconsistent set theory $\mathrm{NF}_{\mathrm{i}}(0 \leq \mathrm{i} \leq \omega)$ and proved that if $\mathrm{NF}_{0}$ is sonsistent, and all Russell's relations (such as $\left\{<\mathrm{x}_{1}, \mathrm{x}_{2}>:<\mathrm{x}_{1}, \mathrm{x}_{2}>\notin \mathrm{x}_{1}\right)$ and $\left\{<\mathrm{x}_{1}, \mathrm{x}_{2}>:<\mathrm{x}_{1}\right.$, $\left.\mathrm{x}_{2}>\notin \mathrm{x}_{2}\right)$ ) exist, then the obtained system is both consistent and adequate. Firstly, in this paper, a definition of the inconsistency and consistency, triviality and non-triviality of the theory $\mathrm{T}$ is given. Secondly, it is pointed out that in his two articles in 1964 and one in 1965, he elaborated a paraconsistent set theory based on the first-order paraconsistent logic, and pointed out that in these systems of set theory, Russell's set existed. Although these systems are non-trivial, they are inconsistent. Moreover, he proved that if the system was non-trivial, then the corresponding classic set theory was consistent. However, a more important result is that the inverse of this theorem holds. Because classical systems are more intuitive and seem to be more reliable than the paraconsistent set theory at first glance. Finally, he emphasized: "In this article, it is proved that if the classic set theory system associated with his system is consistent, then the classic set theory system is extraordinary." Therefore, some inconsistent but extraordinary set theory system is as trustworthy as the standard set theory. In particular, he believed "The set theory system is stronger than the classical set theory [5].

In 1992, G. Restall pointed out in A Note on Naive Set Theory in $L P$, "The recent naive set theory on non-standard logic has many good results." In that article, Restall first introduces the paraconsistent logic LP that he will use, and then formalizes the comprehension axiom model and Axiom of Extension in naive set theory. Second, he proves that this theory is non-trivial and its relationship with ZF. In particular, in that theory, empty sets and universal sets exist. Finally, he has critically evaluated the theory [6]. In 1997, in order to deal with the philosophical problems in paraconsistent set theory, that is, to clarify the epistemological problems between mathematics and empirical science, R. da C. Caiero and E. G. de Souza in A New Paraconsistent Set Theory: ML1, constructed a new paraconsistent set theory $\mathrm{ML}_{1}$ based on Quine's famous set theory system NF and the first-order paraconsistent calculus $\mathrm{C}_{1}{ }^{=}$. They believe that classic set theory cannot prove the existence of the universal set, but the universal set can be deduced from the axioms of $\mathrm{ML}_{1}$, and the entire theory will not fall into non-triviality. In addition, Caiero and de Souza also studied why $\mathrm{ML}_{1}$ can be used as a basis for inconsistent but non-trivial theory. Finally, they have defined the Russell set and studied some of its properties [7].

In the 20th century, the paraconsistent set theory has made great progress. In 2003, T. Libert emphasized ideal calculation in $Z F$ and the Axiom of Choice in Some Paraconsistent Set Theories. Logic and Logical Philosophy, 2003, 11: 91-114. In order to be legal and to keep our concept of naive set safe, and to exclude the paradox that appears in "classical" reasoning, people try to modify the axiom of the existence of sets rather than the basic logic used. This article attempts to find out in a satisfactory way which logic can support these naive (but intuition is correct) concepts. In order to construct such a set theory model, Libert has presented two different techniques. The first one is to modify the classic model of the positive comprehension axiom constructed, and by which the properties gained in the model generate a natural paraconsistent set theory introduced here. In this theory, the axiom of choice is discussed. The second technique leads to the proof the model which is constructed by any classical set 
theory universe (for example, a model of $\mathrm{ZF}$ ) by using corresponding mutual simulation techniques [8]. In 2005, T. Libert pointed out in Models for Paraconsistent Set Theory, "From the Russell paradox we know Cantor's naive set theory based on the first-order axiomatization of the classical logic is inconsistent, while the classical solution to contradictions is to get rid of "contradictions set"". The reason is that these contradictions sets are unnecessary for the basis of mathematics. However, some logicians say that such inconsistent objects can be handled well and have research value in an appropriate theory. For example, non-well-founded sets-universal sets $\mathrm{V}=\{\mathrm{x}: \mathrm{x}=\mathrm{x}\}$ is not essential to the foundation of mathematics, but it is widely used in the field of computer science. There are many examples in mathematics. They introduce "imaginary / ideal" objects, which brings many benefits, but it also forces people to give up some basic properties or principles of mathematics. However, this approach will pay the price. The price to pay mentioned here is that this theory is embedded in logic and may weaken the results in classical reasoning and mathematical practice. Therefore, people may say that doing so outweighs the disadvantages. However, this paper has proves that it is also possible to construct a natural model of paraconsistent set theory. In this article, Libert defines a set theory model as an arbitrary $\in$-structure that satisfies a fragment of the comprehensive axiom model in a given logic. All models should be considered and described in a pre-existing classic global set (for example: a ZF model, if necessary, plus a reasonable large cardinality assumption). In particular, he has also used small $\mathrm{P}$ to indicate the membership function between elements in the metatheory and sets, and a large $\in$ to indicate the membership function in the language of set theory models. In this article, Libert outlines some common techniques for constructing classical models and non-classical models, and has described in detail and applied this technique to construct the model of Paraconsistent Set Theory [9]. In 2006, G. Priest pointed out in Paraconsistent Set Theory. Logic, the problem produced by Russell Paradox and Cantor's Naive Set Theory is generally caused by the following two reasons. First, there exists a principle in an unrestricted comprehension set, which allows that a set can be generated under any conditions. Second, the various principles of logic allow all situations can be included in certain situations (or their connections). Due to the discovery of the paradox, the traditional practice is to maintain logical principles when talking about things, while refusing to accept the comprehension principles without restrictions. The strategy has produced type theory, Zemelo-Frankel set theory, etc. However, there is another possible strategy: sticking to the comprehension principle and reject the logical principle used in some problems. In doing so, there may be many other different ways, but here we are focusing on the way of paraconsistent method. It allows set theory to contain contradictions, and allows $\alpha$ and $\neg \alpha$ to deduce $\beta$, and a theory thus obtained is inconsistent, but not trivial. Therefore, paraconsistent set theory retains the comprehension principles of naive set theory and adopts the set theory of paraconsistent logic [10]. In 2010, Z. Weber in the article Transfinite Numbers in Paraconsistent Set Theory. The Review of Symbolic Logic, 2010, 3 (1): 71-92 holds that the axiom of naive set theory defines the set with the existence and uniqueness conditions. Its first axiom is the comprehension principle. The comprehension principle asserts that the collection of objects is a set and this set is an object of itself. However, this comprehension principle is inconsistent. In the case of classical logic, it can lead to contradictions under the circumstance of the Classical Logic [11]. But for a long time, people have been insisting on classical logic and adopting the method by Zemelo in 1908, and choosing some instances of comprehension principle. But there are also some who insist on the comprehension principle. For example, Routley and Meyer insisted on choosing reasonable logical axioms to deal with it in 1976. In 2012, Z. Weber pointed out in Transfinite Cardinals in Paraconsistent Set Theory, how to understand cardinality in an inconsistent theory is not an easy problem [12]. However, the problem is simple: given Russell set $\mathrm{X}$, then $\mathrm{X} \neq \mathrm{X}$ is inconsistent, so what is the cardinality of unit set $\{x\}$ ? Of course, the answer is " 1 ". Since $X \neq X$, what is the cardinality of the pair $\{\mathrm{X}, \mathrm{X}\}$ composed of two objects? In a proper paraconsistent logic, this paper has developed a (nontrivial) cardinal theory from the comprehensive principle of a naive set. After discussing radix arithmetic, and proving the choice axiom by using the well-ordering theorem, thereby we obtained the result by Zermelo, that is, every set may be well ordered. Regarding Cantor's theorem, Cantor himself gave an indirect proof that suppose there is a surjection of a set to its power set, and then contradictions are obtained, so this assumption cannot be established. However, in a paraconsistent logic, because contradiction is not always absurd, it is not necessarily a reason to reject a hypothesis. Although this is an important challenge, it is not insurmountable. In the paraconsistent logic used in this article, there are many forms to verify the reduction to absurdity. But it is unclear now what kind of reduction to absurdity is used in Cantor's theorem. He used multiple premises, but not the disjunctive syllogism. When deducing a contradiction, there is no general way to pick out the rejected hypothesis. In this article, Weber gives a new proof of Cantor's theorem. Finally, a method to prove the existence of some large cardinal numbers is described by the reflection theorem. In 2013, in Paraconsistent Set Theory by Predicating on Consistency, W. Carnielli and M. E. Coniglio adopted the first-order inconsistent logic (LFIs) and considered consistent and inconsistent statements, as well as consistent and inconsistent sets, and discussed the use of consistent reasoning on the basis of set theory. Under the premise of ZF consistency, they have established the foundation for new paraconsistent set theory (such as ZFmbC and ZFCil) and proved their non-triviality. In order to solve the paradox that appears in the naive set theory, they think that modifying the comprehension rules is only a way of temporary remedy, because (as it's well-known) it is impossible to prove the absolute consistency of set theory. This article uses a fundamental solution, that is, the paraconsistent approach. This approach shows that we do not 
have to solve the paradox. If the consequence relation is paraconsistent, then contradictions will exist, but the contradictions are isolated and cannot be spread to the entire system [13]. In the same year, Weber re-depicted the extensionality principle and set existence principle in Notes on Inconsistent Set Theory. The constructed paracosistent set theory can better accommodate Russell sets and universal sets. In 2014, N. Thomas in Expressive Limitations of Naïve Set Theory, pointed out that Priest studied the set theory based on LP in 2006 [14]. In 1992, Restall first studied the naive set theory on LP, and his theory is different from Priest's [15]. Thomas uses the set theory of Priest version. He gave some negative results based on LP and the naive set theory (NS) on the four variants of $\mathrm{LP}_{\mathrm{m}}, \mathrm{LP}_{=}, \mathrm{LP}_{\subseteq}$ and $\mathrm{LP}_{\supseteq}$ of the minimal discordant logic defined Crabbé in 2011. Thomas has proved that NS is almost trivial in $\mathrm{LP}_{\mathrm{m}}$ and $\mathrm{LP}_{\subseteq}$. In this sense, the only minimal inconsistency model of NS is a 1-meta-model. NS also has the same conclusion on $\mathrm{LP}_{=}$and $\mathrm{LP}_{\supseteq}$. In 2016, W. Carnielli and M. E. Coniglio used Logics of Formal inconsistency (thereafter briefly referred to LFIs) in the paper Paraconsistent Set Theory by Predicating on Consistency to study the consistent-inconsistent statements and the consistent-inconsistent sets, and re-examine the position of contradictory sets in the set history, the authors have developed a new paraconsistent set theory of axiomatization based on the first-order LFIs and admitted that statements and sets can be consistent or inconsistent. The main idea behind LFIs is that contradictions involving negation do not necessarily make a system or theory trivial, but the contradictions within a range are considered to be consistent and will lead to arbitrary conclusions [16]. That is to say, a theory containing a pair of contradictories $\alpha, \neg \alpha$ is unnecessarily not trivial, but any theory containing $\alpha, \neg \alpha$, o $\alpha$ always deduces non-trivialization (o is a link word for consistency in LIFs).

\section{Three Kinds of Logics That Can Be Used to Construct Paraconsistent Set Theory}

In the end of $1970 \mathrm{~s}$, since the introduction of paraconsistent logic to China, there are many of its Chinese translations. Paraconsistent logic is used here for its translation. paraconsistent logic is a logic theory that cannot derive everything from contradictions. But it is the only kind of logic theory that can deal with inconsistencies. Before investigating paraconsistent logic, we first introduce a logical consequence relations and the principle of explosion. The principle of explosion is also often referred to as "Scotus' Law", and its thoughts can be traced back to Aristotle's "Metaphysics". Aristotle believes that there is a certain relationship between the principle of explosion and the law of contradictions that if we admit the principle of explosion, we must admit the law of contradictions; but if we deny the principle of explosion, we cannot certainly yet deduce the law of contradiction [17]. But this conclusion can not be naturally extended to non-classical logic.

For any $\phi$ and $\varphi$, if $\{\phi, \neg \phi\} \vDash \varphi$, then the logical consequence relations $\vDash$ is explosive, $\vDash$ means both semantic consequence and grammatical consequence. According to the definition of the principle of explosion, the consequence relation in classical logic is explosive. A logic is called paraconsistency, iff, its logical consequence relation is not explosive. Therefore, paraconsistent logic is a general term for all logics with paraconsistent property, and not a specific logic.

In logics, there exist many ways to make the logical consequence relation not an explosive one. According to the different ways to achieve paraconsistency, G. Priest divides paraconsistent logic into seven types, of which only three are logics of formal inconsistency (called LFIs for short), multi-valued logic and relevance logic, which can be used to construct paraconsistent set theory [18]. The characteristics of these three types of logics are as follows.

The first type, logics of formal inconsistency (LFIs). LFIs reserve the consistent part of classical logic and only opposes the principle of explosion, namely, the theorem about "for any $\phi$ and $\varphi$, there is $\{\phi, \neg \phi\} \vDash \varphi$ " described in classical logic. In order to achieve this goal, LFIs add the operator of "consistency" to the object language, and puts the meta-theoretical concepts of "consistency" and "inconsistency" into the object language. Therefore, in LFIs, when "consistency" operator appears, its reasoning is the same as that of classical logic. When "consistency" operator does not appear, its reasoning does not recognize the principle of explosion.

The second type, the relevant logics. The definition of conditional sentence is different between relevant logics and classical logic. The former uses "relevant implication", while the latter "substantial implication". In the relevant implication, the conclusion of reasoning is required to be relevant with the premise, so it is set at the beginning that if $\phi$ is relevant with implication $\varphi$, then $\phi$ and $\varphi$ have at least one common proposition variable. The setting of relevant implication invalidates the principle of explosion, so this kind of relevant logic is paraconsistent logic, but not all of them are paraconsistent logic. In the material implication, the principle of explosion is valid.

The third type, the multi-valued logic. Multi-valued logic allows propositions to have truth values other than true and false. Suppose that $\mathrm{c}$ is the third true value except true and false. If $\phi$ is true and "non- $\phi$ " is false, then the truth value of " $\phi$ and non" is c. In multi-valued logic concerning the setting of conditional sentences, the proposition with value $\mathrm{c}$ does not imply any proposition, and the principle of explosion is made invalid.

\section{The Strength and Weakness of Paraconsistent Logic}

As an important branch of philosophical logic which has been deeply and extensively studied, paraconsistent logic has 
its strength and weakness, and has a certain relationship with classical logic. The consistency of classical logic is not included in the strong paraconsistent logic, but in the weak paraconsistent logic. Different methods are used to construct the paraconsistent set theory with strong or weak paraconsistent logic, and the resulting paraconsistent set theory is not the same. Based on the strong paraconsistent logic, the paraconsistent naïve set theory can be constructed. Since the consistency part of classical logic is not included in the strong paraconsistent logic, if we want to construct strong set theory, we need to use the axioms of strong set theory, such as comprehension axioms and extension axioms. Based on the weak paraconsistent logic, and according to the different expectations of the paraconsistent theory to the paraconsistent set theory, only by replacing the basic logic of naïve set theory, ZF, NBG (set theories by von· Neumann, Godel, and Benas), and the basic logic of NF, can we construct the set theory stronger than naïve set theory, ZF, NBG, and NF, such as, paraconsistent naïve set theory, paraconsistent $Z F$, paraconsistent NBG, and etc. According to Priest's research, any acceptable paraconsistent set theory must satisfy the two conditions: first, it cannot be trivial; second, its statements about set should be more than that of ZF. The set theory mentioned here is axiomatic set theory. Its structure can be divided into basic logic (i.e. classical logic) and set-theory axioms. In general, if logic $\mathrm{C}$ contains logic $\mathrm{D}$ when axiom of set theory is invariable, then the set theory based on the construction of logic $\mathrm{C}$ is stronger than set theory based on that of logic D. the "strong" here refers to the more content stated about set [19].

LFIs is a weak paraconsistent logic, and its typical system is Costa's $C_{n}(1 \leq n<\omega)$ system (excluding $\left.C_{\omega}\right)$. Subsequently, Costa and Oruda constructed the paraconsistent NF set theory NFn on the basis of the $C_{n}(1 \leq n<\omega)$ system. In 1997, R. da C. Caiero and E. G. de Souza constructed a paraconsistent set theory $\mathrm{ML}_{1}$ based on the extension $\mathrm{ML}$ system and paraconsistent $\operatorname{logic} \mathrm{C}=1$ of NF. In addition to the $\mathrm{C}_{\mathrm{n}}$ system, there is the weak paraconsistent logic mbc, which is one of the LFIs [20]. In 2013, W. Carnielli and M. E. Coniglio constructed the paraconsistent set theory ZFCil based on mbc and ZF [21] [22].

The constructed strong paraconsistent logic system includes: the typical system in relevance logic, which is the systems of DM and DL by R. Routley and R. K. Meyer. Then, Z. Weber established the paraconsistent logic TLQ and TKQ based on Routley's ideas. In multi-valued logic, LP system constructed by Priest in 1979 was also a strong paraconsistent logic [23].

\section{A New Solution to the Paradox of Set Theory Pradox}

Facing the contradictory problem, the solution of the paraconsistent set theory is not the same as the previous common practice. paraconsistent logic is to limit the scope of application of the law of contradictions in various ways. If a logic system restricts the law of contradictions, then the system belongs to paraconsistent logic. There are many ways to express the law of contradictions, such as (1) One is a false in two propositions of mutual negation. (2) $\neg(\phi \wedge \neg \phi)$. (3) The same individual cannot have both a property and that property itself at the same time [19]. In the past, dealing with set theory paradoxes was often to eliminate contradictions in order to ensure the consistency of the set systems. While the goal of the paraconsistent set theory in dealing with set theory paradoxes is to tolerate contradictions, accept the inconsistency of set theory systems, and ensure the non-triviality of the set theory systems. In the view of paraconsistent arguers, the real reason for the loss of the research significance of the naive set theory is not its inconsistency, but its triviality. The paraconsistent set theory is a new tool to solve the paradoxes of the set theory.

The principle of explosion is a theorem in classical logic. Any logical system that does not recognize the effective form $\{\phi, \neg \phi\} \vDash \varphi$ in classical logic belongs to the paraconsistent logic system. Assuming that the explosion principle is valid, for any $\phi$ and $\varphi,\{\phi, \neg \phi\} \vDash \varphi$, it is shown that the inconsistency and triviality are equivalent. In the naive set theory based on classical logic, the contradiction arising means that the naive set theory is trivial. Suppose further that the explosion principle fails, when there exist $\phi$ and $\varphi$, there comes $\{\phi, \neg \phi\} \not \models \varphi$, indicating that inconsistency does not necessarily lead to triviality. paraconsistent logic does not recognize the validity of the explosion principle. Therefore, the appearance of contradictions in the paraconsistent set theory based on the paraconsistent logic does not mean that the paraconsistent set theory is trivial, and the paraconsistent set theory is an inconsistent but non-trivial theory. Therefore, non-triviality is the criterion for judging whether a paraconsistent set theory can successfully handle set theory paradox. If a paraconsistent set theory is non-trivial, it can successfully deal with set theory paradoxes. With non-triviality as the standard, all paraconsistent set theory can successfully deal with Russell paradox. Because the multi-valued logic LP system of paraconsistent logic does not recognize the validity of MP, which makes the paraconsistent naive set theory based on LP construction free from the equivalence paradox [3]. The relevance logic, as a paraconsistent logic, does not recognize the validity of the contraction law, which makes the paraconsistent naïve set theory based on relevance logic avoid the triviality caused by the equivalent paradox. Therefore, the paraconsistent set theory will better deal with the paradoxes of set theory.

\section{Conclusion}

Naive set theory is the foundation of modern mathematics and an important branch of modern logic. The "Russell Paradox" that appeared in the naive set theory directly affects the development of modern mathematics and modern logic, and also has a profound impact on the development of set theory. Classic paradox solutions cannot accommodate such contradiction sets, because these sets are unnecessary for the foundations of mathematics, and the set theory as the 
foundations of mathematics requires the consistency of sets; some logicians insist on adjusting the basic logic of these theories can deal with and study similar inconsistent objects, and thus obtain the paraconsistent theory. As a new method to deal with the paraconsistent set theory, the unique theory of set theory is the attitude towards contradiction and standard of successfully dealing with the paradox of set theory. The attitude towards contradiction is tolerance rather than elimination. The standard of successful handling of the paradox of set theory is to ensure its own nontrivial.

The development of the paraconsistent set theory can solve the difficulties encountered in the development of set theory in a unique way. This is not only the promotion of the application of paraconsistent logic, but also the new form and new trend of the development of set theory. More than that, the thoughts and methods in paraconsistent set theory can promote the development of paraconsistent arithmetic and inconsistent mathematics, and thus advance the entire process of the inconsistent theoretical research.

\section{References}

[1] Li Na, "Research on Set Theory Based on Philosophical Logic". Journal of Zhejiang University (Humanities and Social Sciences), 2017 (1): 215-216.

[2] A. I. Arruda, da Costa. "A hierarchy of formal systems", C. R. Acad. Sc. Paris, 259, 1964, 2943-2945.

[3] A. I. Arruda, D. Batens, "Russell's set versus the universal set in paraconsistent set theory". Logique et Analyse, 1982 (98): 121-133.

[4] A. Arruda, "Remarks on da Costa's paraconsistent set theories", Rev Colombiana Mat, 1985, 19 (1-2): 9-24.

[5] G. Restall, "A Note on Naive Set Theory in LP", Notre Dame Journal of Formal Logic, 1992, 33 (3): 422-432.

[6] Da Costa, "On paraconsistent set theory". Logique et Analyse, 1986, 29 (115): 361-371.

[7] R. da C. Caiero, E. G. de Souza, "A New Paraconsistent Set Theory: ML1", Logique \& Analyse, 1997, 157: 115-141.

[8] T. Libert, "ZF and the Axiom of Choice in Some Paraconsistent Set Theories", Logic and Logical Philosophy, 2003, 11: 91-114.

[9] T. Libert, "Models for Paraconsistent Set Theory", Journal of Applied Logic, 3, 15-41.

[10] G. Priest, "Paraconsistent Set Theory. Logic", Mathematics, Philosophy, Vintage Enthusiasms. Springer Netherlands, 2011, 153-169.
[11] Zach Weber. "Transfinite numbers in paraconsistent set theory". The Review of Symbolic Logic, 2010, 3 (1): 71-73.

[12] Zach Weber. "Transfinite cardinals in paraconsistent set theory". The Review of Symbolic Logic, 2012, 5 (2): 269-293.

[13] W. Carnielli, M. E. Coniglioin, "Paraconsistent Set Theory by Predicating on Consistency", Journal of Logic and Computation, 2013.

[14] Nick Thomas. "Expressive limitations of naive set theory in LP and minimally inconsistent LP". The Review of Symbolic Logic, 2014, 7 (2): 341-350.

[15] Greg Restall. "A note on naive set theory in LP". Notre Dame Journal of Formal Logic, 1992, 33 (3).

[16] Walter Carnielli and Marcelo E. Coniglio. "Paraconsistent set theory by predicating on consistency". Journal of Logic and Computation, 2016, 26 (1): 97-116.

[17] Zhang Jianjun, "Editor's Guide: A few questions about paraconsistent logic", Logic Research, 2018 (2): 1-8.

[18] Li Na, He Jianfeng, "A New Method to Deal with the Paradox of Set Theory”, Philosophical Trends, 2017 (11): 95-97.

[19] Zhang Qingyu, "paraconsistent logic", China Society Press, Beijing, 2003.: 23.

[20] W. Carnielli, M. E. Coniglio, J. Marcos, "Logics of Formal Inconsistency", Hankbook of Philosophical Logic, Vol. 14, Springer, 2007: pp 1-94.

[21] W. Carnielli, M. E. Coniglio, "Paraconsistent Set Theory By Predicating on Consistency", Journal of Logic and Computation, 26 (1), 2013: 1-20.

[22] G. Priest, "The Logic of Paradox", Journal of Philosophical Logic, 8 (1), 1979, pp. 219-241.

[23] Zhang Qingyu, "paraconsistent logic, An Exploration of Logic", 1996 (11): 18.

\section{Biography}

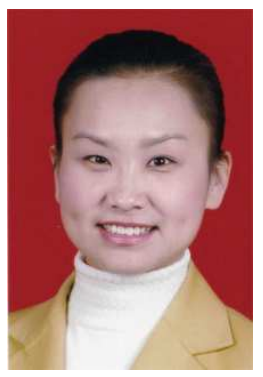

Shi Jing, female, was born in 1980, in Zhengzhou City, Henan Province, China. Since 2003, she had been studying modern logic with Professor $\mathrm{Na} \mathrm{Li}$, a famous logician in China. In 2009, she graduated from the school of philosophy of Nankai University with a Ph.D. and is now an associate professor of the School of Culture and Media of the Central University of Finance and Economics and a Master's Supervisor of Logic. Her main study field is in symbolic logic and philosophical Logic. 